\title{
Der neue Fähigkeitsausweis POCUS
}

\author{
Christoph Knoblauch ${ }^{a}$, Corina Canova ${ }^{b}$, Werner Bauer ${ }^{c}$, Christoph Ganter ${ }^{d}$ \\ a Präsident Weiterbildungskommission (WBK) POCUS; ${ }^{b}$ Präsidentin SGUM; ${ }^{c}$ Präsident SIWF; d Präsident ICAN und Vizepräsident WBK POCUS
}

\section{Historie}

Die Ultraschalltechnik wird seit bald 100 Jahren für medizinische Zwecke angewandt. Die ersten farbkodierten Darstellungen hielten in den 80er Jahren mit der Verfügbarkeit von leistungsstarken Rechnern Einzug. 1969 entstand die Schweizerische Arbeitsgemeinschaft für Ultraschall (SAGU) aus einer Gruppe begeisterter Ultraschaller. Die SGUM/SSUM (Schweizerische Gesellschaft für Ultraschall in der Medizin) besteht seit 1980 (hiess damals SGUMB/SSUMB: Schweizerische Gesellschaft für Ultraschall in der Medizin und Biologie) und wurde von der SAGU an einer ersten konstituierenden Sitzung in Zürich gegründet.

In der Schweiz hat sich die Schweizerische Gesellschaft für Ultraschall in der Medizin (SGUM) der Aus- und Weiterbildung verpflichtet (Abb. 1).

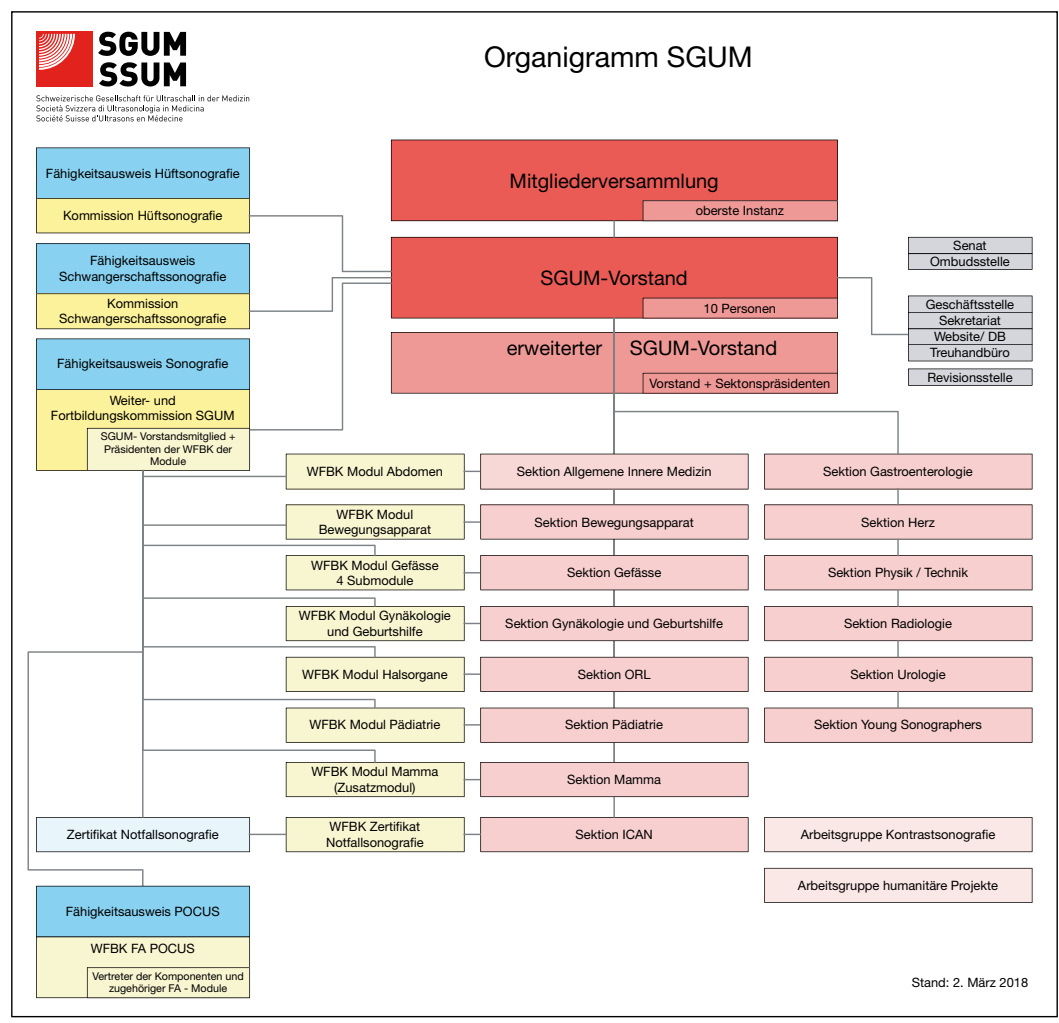

SGUM Organigramm
Ursprünglich galt als eisernes Prinzip, dass die ein zelne Untersuchung nur durch klar definierte und hochqualifizierte Spezialisten durchgeführt werden konnte. Zudem musste die Untersuchung eine ganze anatomische Region, eine physiologische Einheit oder ein Organ umfassen. Es mussten alle in Frage kommenden Pathologien überprüft werden, was lange Untersuchungszeiten mit sich brachte.

\section{Die nicht invasive bildgebende Untersuchungs- methode hat sich in der Breite und auch in der Aufgabenstellung verändert.}

Die nicht invasive bildgebende Untersuchungsmethode hat sich seither in der Breite und auch in der Aufgabenstellung verändert und ist heute als Bedside-Methode verfügbar. Spezifische Fragestellungen können durch die Point of care-Ultraschallmethode fokussierter und effizienter beantwortet werden. Dabei steht die Übersetzung klinischer Probleme in einfache, sonographische Ja/Nein-Fragestellungen im Vordergrund. Entsprechend hat sich die Schaffung eines Fähigkeitsausweises POCUS mit aktuell 14 definierten Komponenten aufgedrängt. Er wird ab dem kommenden Jahr in der klinischen Praxis eingeführt (Tab.1).

\section{Fähigkeitsausweis POCUS und seine Komponenten}

Der Fähigkeitsausweis (FA) POCUS ermöglicht gezielte Untersuchungen, die eine konkrete Fragestellung beantworten können, und erlaubt eine ultraschallgesteuerte Intervention mit grossen Vorteilen gegenüber dem konventionellen Vorgehen (schneller, sicherer und effizienter). 2013 wurde in der SGUM die Sektion ICAN (Intensivmedizin, Chirurgie, Anästhesie und Notfallmedizin) gegründet. Sie initiierte das Modell von fokussierten Komponenten mit dem Ziel, eine strukturierte Aus-, Weiter- und Fortbildung für diese Ultraschallgebiete aufzubauen. Bereits kurz vorher leisteten die Notfallmediziner erste Pionierarbeit mit dem Aufbau des Zertifikats Notfallsonographie.

Mit der Zeit ist durch eine intensive interdisziplinär ge- 
Tabelle 1: Komponenten.

Basis-Notfall-Sonographie

Fokussierte Sonographie am Bewegungsapparat

Fokussierte Sonographie arterielle Gefässe

Fokussierte Sonographie venöse Gefässe (inklusive endovenöse Therapie

oberflächlicher Venen)

Gefässpunktionen

Interventionelle Schmerztherapie

IPS für Kinder ab 5. Lebenswoche (Grundlagen, Thorax, Abdomen, Gefässe)

Neuro-Critical Care

Pädiatrie Praxis / Notfallstation / Ambulatorium

Regionalanästhesie

Fokussierte Thoraxsonographie

Fokussierte transösophageale kardiale Sonographie (Erwachsene auf Intensivstation,

im Operationssaal und in Notfallsituationen)

Fokussierte transthorakale kardiale Sonographie (Erwachsene auf Intensivstation,

im Operationssaal und in Notfallsituationen)

Fokussierte transthorakale kardiale Sonographie (Kinder ab 5. Lebenswoche auf

Intensivstation, im Operationssaal und in Notfallsituationen)
Korrespondenz:

Dr. med. Christoph

Knoblauch

Chefarzt Medizin

Kantonsspital Nidwalden

christoph.knoblauch[at]

ksnw.ch führte Diskussion die Erkenntnis gewachsen, dass ein gemeinsamer Fähigkeitsausweis POCUS den verschiedenen Anliegen unterschiedlicher Sektionen am meisten dienen wird. Erarbeitet wurde das Konzept eines komponentenartig aufgebauten Fähigkeitsausweises. Dieser kann zukünftigen Entwicklungen flexibel Rechnung tragen und ermöglicht den Einbezug sonographierender Facharztgesellschaften.

Parallel dazu setzte sich in der Weiterbildungskommission der Schweizerischen Gesellschaft für Allgemeine Innere Medizin der Wunsch durch, die Ultraschalltechnik fix in das E-Logbuch der Auszubildenden zu integrieren. Gleichzeitig bestehen Bestrebungen der Universitäten, die Sonographie bereits im Studium als Lernziel einzusetzen.

Damit entstand in Zusammenarbeit mit dem SIWF der vorliegende Fähigkeitsausweis POCUS, im Wissen, dass damit eine Zwischenlösung erarbeitet wurde, bevor die Ultraschalltechnik zu einem späteren Zeitpunkt als fixer Ausbildungsbestandteil der Ärzteschaft Eingang in die Logbücher findet.

Um die administrativen Voraussetzungen zur Implementierung des neuen Fähigkeitsausweises POCUS zu garantieren, hat die SGUM erstens eine separate Weiterbildungskommission POCUS ins Leben gerufen und zweitens die Geschäftsstelle umstrukturiert und professionalisiert. Neu ist mit Meister ConCept $\mathrm{GmbH}$ eine im Verbandsmanagement erfahrene Organisation für die Belange der SGUM verantwortlich. Die direkte Ansprechperson für die SGUM ist Frau Fabienne Lüthi (sgum[at]sgum.ch). Die Abwicklung der Fähigkeitsausweise wird in Zukunft über eine eigens für die SGUM erstellte Datenbank elektronisch erfolgen.
Die Erarbeitung der Lernziele für die jeweiligen Komponenten war sehr anspruchsvoll, zumal verschiedene Interessensvertreter einen gemeinsamen Weg finden mussten. Es gelang aber letztlich, sämtliche Fachexperten von der Notwendigkeit eines gemeinsamen Weges zu überzeugen und ins Boot zu holen. Die aktuelle Version stellt den gefundenen Kompromiss dar. Notwendige Anpassungen wird uns der Alltag zeigen Wir werden diese konsequent interdisziplinär unter Einbezug der Facharztgesellschaften umsetzen.

So sind heute nebst den bereits mit der SGUM zusammenarbeitenden Spezialitäten neue und wichtige Player wie die Kardiologen und die Pneumologen mitbeteiligt. Der detaillierte Beschrieb des Fähigkeitsprogramms Point of Care-Ultraschall POCUS und die einzelnen Komponenten mit den Lernzielen können der Website der SGUM entnommen werden.

\section{Zukunftsaussichten}

$\mathrm{Ab}$ sofort wird die Weiterbildungskommission POCUS die offenen Fragestellungen wie Übergangsregelungen, die Bereinigung der Datenbank mit Alt- und Neumitgliedern und die verschiedenen Berechtigungen (Tutoren, Kursleiter) definieren. Gleichzeitig gilt es die bestehenden Kurse an die neuen Lernziele anzupassen und für den FA POCUS anzuerkennen. Hierfür sind wiederum die Komponentenvertreter zuständig. Speziell in der Anfangsphase werden pragmatische Lösungen angestrebt. Wie POCUS in Zukunft organisatorisch in die SGUM eingeordnet wird, ist noch Gegenstand der Diskussion und wird später entschieden.

Selbstverständlich ist damit die Entwicklung im Bereich Ultraschalldiagnostik der Medizin nicht abgeschlossen. Nicht nur die Schweizerische Gesellschaft für Allgemeine Innere Medizin (SGAIM) möchte die Ultraschallausbildung im Curriculum verankert sehen. Wie bereits erwähnt, sind es heute die Universitäten, welche die Methodik des Ultraschalls propagieren und verschiedene Kurse zum Kennenlernen der Anatomie von Gewebe und Organen ins Leben rufen. Hier lässt beispielsweise das Projekt des blended e-learning Grundkurs Abdomensonographie der Sektion Young Sonographers aufhorchen. Dieser Kurs birgt das Potential, die Grundlagen der Ultraschalldiagnostik in modularer Form bereits während des Studiums allen zugänglich zu machen, und wird zurzeit zusammen mit SGUM-Vertretern bearbeitet. Er könnte künftig stellvertretend für den theoretischen Teil des Grundkurses Abdomen gelten. Bis wir so weit sind, wird allerdings noch Zeit vergehen.

\section{Literatur}

Osterwalder J. Das SGUM-Zertifikat Notfallsonographie. Schweiz Ärztezeitung. 2012;93(34):1210-1. 